\title{
On the Distribution of First Exit Time for Brownian Motion with Double Linear Time-Dependent Barriers
}

\author{
Lin Xu and Dongjin Zhu \\ School of Mathematics and Computer Science, Anhui Normal University, Wuhu 241000, China \\ Correspondence should be addressed to Dongjin Zhu; djzhu@mail.ahnu.edu.cn
}

Received 2 July 2013; Accepted 28 August 2013

Academic Editors: G. Kyriacou and C. Lu

Copyright (C) 2013 L. Xu and D. Zhu. This is an open access article distributed under the Creative Commons Attribution License, which permits unrestricted use, distribution, and reproduction in any medium, provided the original work is properly cited.

\begin{abstract}
This paper focuses on the first exit time for a Brownian motion with a double linear time-dependent barrier specified by $y=a+b t$, $y=c t,(a>0, b<0, c>0)$. We are concerned in this paper with the distribution of the Brownian motion hitting the upper barrier before hitting the lower linear barrier. The main method we applied here is the Girsanov transform formula. As a result, we expressed the density of such exit time in terms of a finite series. This result principally provides us an analytical expression for the distribution of the aforementioned exit time and an easy way to compute the distribution of first exit time numerically.
\end{abstract}

\section{Introduction}

In financial investment affairs, investors are exposed to credit risk, due to the possibility that one or more counterparts in a financial agreement will default (cf [1]). The default time is sometimes modeled as the first exit time of a credit index process below a barrier. Original credit models can be found in Merton [2] and Black and Cox [3], where the return of market value is supposed to be a drifted Brownian motion. When the market value of assets goes below some level, determined in terms of the company's liabilities, then the company is apt to default on its obligations. The first passage time density is required in order to obtain the expected discounted cash flows (say for a loan to the considered company). They define the time of default as the first time the ratio of the value of a firm and the value of its debt falls below a constant level, and they model debt as a zero-coupon bond and the value of the firm as a geometric Brownian motion. In this case, the default time has the distribution of the first-passage time of a Brownian motion (with constant drift) below a certain barrier. Hull and White [4] model the default time as the first time a Brownian motion hits a given time-dependent barrier. They show that this model gives the correct market credit default swap and bond prices if the time-dependent barrier is chosen so that the first passage time of the Brownian motion has a certain distribution derived from those prices. Given a distribution for the default time, it is usually impossible to find a closed-form expression for the corresponding time-dependent barrier, in derivatives pricing, such as pricing barrier options or lookback options, which involve crossing certain levels (cf Chadam et al. [5], Merton [6], and Metwally and Atiya [7]), or pricing American options [8], which entail evaluating the first passage time density for a time varying boundary. Such applications are typically applied to large portfolios involving thousands of securities, and, in addition, thousands of iteration runs are needed on each security in the portfolio to calibrate the model parameters. With the developments of financial derivatives, all kinds of model related to Brownian motion arose and consequently the distribution of first exit time for Brownian motion has attracted more and more attention recently (cf [9-11]).

In this paper, we consider the case that the investor has the chance to receive dividend barrier once the surplus of investor's wealth goes beyond a given linear timedependent barrier and taking the risk of default at the same time. Such policy makes the products more attractive for investors. Standing on the point of investors, what they are concerned with is the probability of the return process hitting 
the dividend barrier before hitting the default barrier. Thus, our problem can be formulated in the first exit time for Brownian motion with double linear time dependent barrier (for barrier for describing the dividend barrier and the other for default barrier).

Usually, there is no analytic solution or close form expression for distribution (or density) of the first exit time of Brownian motion other than the case of singer linear timedependent barrier. As an alternative, many works fall back on estimation or approximating the distribution or density of first exit time of Brownian motion (cf [11-15]). In this paper, by applying Girsanov theorem iteratively, we obtained the density of first exit time for Brownian motion from a double linear time-dependent barrier in terms of infinity series. This result provides us a convenient way to compute the density function numerically. This paper is organized as follows. Section 2 briefly introduces some preliminary results on Brownian motion and the problem to be investigated. Section 3 provides the methods and results on the problem studied in this paper.

\section{Preliminaries and Problem to Be Investigated}

Definition 1. Stochastic process $\{W(t), t \geq 0\}$ is a Brownian motion in thefollowing conditions

(i) $W(0)=0$, a convenient normalization.

(ii) Independent increments: whenever $0=t_{0}<t_{1}<\cdots$ $<t_{n}$

$$
W_{t_{1}}-W_{t_{0}}, \ldots, W_{t_{n}}-W_{t_{n-1}} \text { are independent. }
$$

(iii) Stationary increments: the distribution of $W_{t}-W_{s}$ only depends on $t-s$ and $W_{t} \sim N(0, t)$.

(iv) $t \rightarrow W_{t}$ is continuous.

The following Lemmas serve as a quick review of the results on Girsanov transform; see Oksendal [16].

Lemma 2 (Girsanov theorem, cf [17] or [16]). Let $Y(t) \in R^{n}$ be an Itô process of the form

$$
d Y(t)=\beta(t, \omega) d t+\theta(t, \omega) d W_{t} ; \quad t \leq T,
$$

where $W_{t} \in R^{m}, \beta(t, \omega) \in R^{n}$, and $\theta(t, \omega) \in R^{n \times m}$. Suppose that there exist $u(t, \omega) \in R^{n}$ and $\alpha(t, \omega) \in R^{n}$ and assume that

$$
\theta(t, \omega) u(t, \omega)=\beta(t, \omega)-\alpha(t, \omega)
$$

and assume that $u(t, \omega)$ satisfies Novikov's condition

$$
E\left[\exp \left(\frac{1}{2} \int_{0}^{T} u^{2}(t, \omega)\right)\right]<\infty .
$$

Put

$$
\begin{gathered}
M_{t}=\exp \left(-\int_{0}^{t} u(s, \omega) d W_{s}-\frac{1}{2} \int_{0}^{t} u^{2}(s, \omega) d s\right) ; \quad t \leq T \\
d Q(\omega)=M_{T}(\omega) d P(\omega) \text { on } F_{T} .
\end{gathered}
$$

Then

$$
\widehat{W}_{t}=\int_{0}^{t} u(s, \omega)+W_{t} ; \quad t \leq T
$$

is a Brownian motion with respect to $Q$.

Let $\left\{W_{t}+x\right\}$ be standard BM with $W_{0}=x$, where $x \in$ $(0, a), b, c$ are positive real number,

$$
\begin{gathered}
T_{1}=\inf \left\{t, W_{t}+x>a-b t\right\}, \\
T_{2}=\inf \left\{t, W_{t}+x<c t\right\} .
\end{gathered}
$$

The purpose of this paper is to estimate

$$
\mathbb{P}_{x}\left(T_{1} \in d t, T_{1}<T_{2}\right), \quad E_{x}\left[T_{1} I_{\left[T_{1}<T_{2}\right]}\right],
$$

where we adopt the symbol $\mathbb{P}\left(\cdot \mid W_{0}=x\right), \mathbb{E}_{x}[\cdot]=\mathbb{E}\left[\cdot \mid W_{0}=\right.$ $x]$.

\section{Distribution of First Exit Time of BM from Double Linear Barrier}

$$
\begin{aligned}
& \text { Denote } X_{t}=x+W_{t}-c t \text {; then } \\
& \qquad \begin{array}{c}
T_{1}=\inf \left\{t, X_{t}>a-(b+c) t\right\}, \\
T_{2}=\inf \left\{t, X_{t}<0\right\} .
\end{array}
\end{aligned}
$$

Denote by $Q_{1}$ the probability measure such that $X_{t}$ is a BM; then by Girsanov theorem we know that

$$
\mathbb{E}\left[\frac{d \mathbb{P}_{x}}{d Q_{1}} \mid F_{t}\right]=\exp \left\{-c\left(X_{t}-x\right)-\frac{c^{2}}{2} t\right\} .
$$

Denote

$$
\begin{gathered}
T_{3}=\inf \left\{t, X_{t}<-a+(b+c) t\right\}, \\
T_{4}=\inf \left\{t>T_{2}, X_{t}>a-(b+c) t\right\} .
\end{gathered}
$$

Then

$$
\begin{aligned}
& Q_{1}\left[T_{1} \in d t\right]=\frac{a-x}{\sqrt{2 \pi t^{3}}} \exp \left\{-\frac{(a-x-(b+c) t)^{2}}{2 t}\right\} d t, \\
& Q_{1}\left[T_{3} \in d t\right]=\frac{a+x}{\sqrt{2 \pi t^{3}}} \exp \left\{-\frac{(a+x-(b+c) t)^{2}}{2 t}\right\} d t .
\end{aligned}
$$

By reflection principle for $\mathrm{BM}$ we have

$$
\begin{aligned}
Q_{1} & {\left[T_{1} \in d t, T_{1}<T_{2}\right]-Q_{1}\left[T_{1}<T_{2}, T_{4} \in d t\right] } \\
= & Q_{1}\left[T_{1} \in d t, T_{1}<T_{2}\right]-Q_{1}\left[T_{1}<T_{2}, T_{3} \in d t\right] \\
= & Q_{1}\left[T_{1} \in d t\right]-Q_{1}\left[T_{3} \in d t\right] \\
= & {\left[\frac{a-x}{\sqrt{2 \pi t^{3}}} \exp \left\{-\frac{(a-x-(b+c) t)^{2}}{2 t}\right\}\right.} \\
& \left.-\frac{a+x}{\sqrt{2 \pi t^{3}}} \exp \left\{-\frac{(a+x-(b+c) t)^{2}}{2 t}\right\}\right] d t .
\end{aligned}
$$


Note that with the symmetric property of $X_{t}$ we have

$$
\begin{aligned}
& \mathbb{P}_{x}\left[T_{1}<T_{2}, T_{4} \in d t\right] \\
&= Q_{1}\left[T_{1}<T_{2}, T_{4} \in d t\right] \\
& \times \exp \left\{-c(a-(b+c) t-x)-\frac{c^{2}}{2}\right\} \\
&= Q_{1}\left[T_{1}<T_{3}, T_{3} \in d t\right] \\
& \times \exp \left\{-c(a-(b+c) t-x)-\frac{c^{2}}{2}\right\} .
\end{aligned}
$$

If we let $Q_{2}$ denote the probability measure such that $W^{(1)}+$ $x=X_{t}+(b+c) t$ is a $\mathrm{BM}$, then

$$
\begin{aligned}
Q_{1}[ & \left.T_{1}<T_{3}, T_{3} \in d t\right] \\
= & \exp \left\{-(b+c)(-a-x+2(b+c) t)-\frac{1}{2}(b+c)^{2} t\right\} \\
& \times Q_{2}\left[T_{1}<T_{3}, T_{3} \in d t\right] \\
= & \exp \left\{-(b+c)(-a-x+2(b+c) t)-\frac{1}{2}(b+c)^{2} t\right\} \\
& \times \mathbb{P}_{x}\left[T_{5}<T_{6}, T_{5} \in d t\right],
\end{aligned}
$$

where

$$
\begin{aligned}
& T_{5}=\inf \left\{t, X_{t}>3 a-2(b+c) t\right\}, \\
& T_{6}=\inf \left\{t, X_{t}<-a+2(b+c) t\right\} .
\end{aligned}
$$

Denote $X_{t}^{(1)}=x+a+W_{t}$ and define

$$
\begin{aligned}
& T_{1}^{(1)}=\inf \left\{t, X_{t}^{(1)}>4 a-2(b+c) t\right\}, \\
& T_{2}^{(1)}=\inf \left\{t, X_{t}^{(1)}<2(b+c) t\right\}, \\
& \mathbb{P}_{x}\left[T_{5}<T_{6}, T_{5} \in d t\right] \\
& =\mathbb{P}_{a+x}\left[T_{1}^{(1)}<T_{2}^{(1)}, T_{1}^{(1)} \in d t\right] .
\end{aligned}
$$

Thus

$$
\begin{aligned}
\mathbb{P}_{x}[ & \left.T_{1}<T_{3}, T_{3} \in d t\right] \\
= & \exp \left\{-(b+c)(-a-x+2(b+c) t)-\frac{1}{2}(b+c)^{2} t\right\} \\
& \times \exp \left\{-c(a-(b+c) t-x)-\frac{c^{2}}{2} t\right\}, \\
\mathbb{P}_{a+x} & {\left[T_{1}^{(1)}<T_{2}^{(1)}, T_{1}^{(1)} \in d t\right] } \\
= & \exp \left\{a b-x b-4 c b t+2 c x-\frac{5 b^{2}+4 c^{2}}{2} t\right\} \\
& \times \mathbb{P}_{a+x}\left[T_{1}^{(1)}<T_{2}^{(1)}, T_{1}^{(1)} \in d t\right] .
\end{aligned}
$$

Repeating previous steps, it follows that

$$
\begin{aligned}
& \mathbb{P}_{a+x}\left[T_{1}^{(1)}<T_{2}{ }^{(1)}, T_{1}{ }^{(1)} \in d t\right] \\
& =\left[\frac{3 a-x}{\sqrt{2 \pi t^{3}}} \exp \left\{-\frac{(3 a-x-4(b+c) t)^{2}}{2 t}\right\}\right. \\
& \left.\quad-\frac{5 a+x}{\sqrt{2 \pi t^{3}}} \exp \left\{-\frac{(5 a+x-4(b+c) t)^{2}}{2 t}\right\}\right] \\
& \times \exp \left\{-2(b+c)(3 a-4(b+c) t-x)-\frac{4(b+c)^{2}}{2} t\right\} d t \\
& \times \exp \{8 a(b+c)+2(a+x)(b+c) \\
& \quad \times \mathbb{P}_{5 a+x}\left[T_{1}^{(2)}<T_{2}^{(2)}, T_{1}^{(2)} \in d t\right] .
\end{aligned}
$$

Wherein,

$$
\begin{gathered}
T_{1}^{(2)}=\inf \left\{t, x+5 a+W_{t}>16 a-8(b+c) t\right\}, \\
T_{2}^{(2)}=\inf \left\{t, x+5 a+W_{t}<8(b+c) t\right\} .
\end{gathered}
$$

By an inductive method, we have

$$
\begin{aligned}
& \mathbb{P}_{x}\left[T_{1}<T_{3}, T_{1} \in d t\right] \\
& =\left[\frac{a-x}{\sqrt{2 \pi t^{3}}} \exp \left\{-\frac{(a-x-(b+c) t)^{2}}{2 t}\right\}\right. \\
& \left.\quad-\frac{a+x}{\sqrt{2 \pi t^{3}}} \exp \left\{-\frac{(a+x-(b+c) t)^{2}}{2 t}\right\}\right] \\
& \times \exp \left\{-c(a-(b+c) t-x)-\frac{c^{2}}{2} t\right\} d t \\
& +\left[\frac{3 a-x}{\sqrt{2 \pi t^{3}}} \exp \left\{-\frac{(3 a-x-4(b+c) t)^{2}}{2 t}\right\}\right. \\
& \left.\quad-\frac{5 a+x}{\sqrt{2 \pi t^{3}}} \exp \left\{-\frac{(5 a+x-4(b+c) t)^{2}}{2 t}\right\}\right] \\
& \times \exp \left\{a b+x b-4 c b t+2 c x-\frac{5 b^{2}+4 c^{2}}{2} t\right\} d t \\
& \quad \exp \left\{-2(b+c)(3 a-4(b+c) t-x)-\frac{4(b+c)^{2}}{2} t\right\}
\end{aligned}
$$




$$
\begin{aligned}
& +\sum_{k=2}^{\infty}\left[\frac{4^{k} a-\sum_{j=1}^{k-1} 4^{j} a-x}{\sqrt{2 \pi t^{3}}}\right. \\
& \times \exp \left\{-\frac{\left(4^{k} a-\sum_{j=1}^{k-1} 4^{j} a-x-4^{k}(b+c) t\right)^{2}}{2 t}\right\} \\
& -\frac{4^{k} a+\sum_{j=1}^{k-1} 4^{j} a+x}{\sqrt{2 \pi t^{3}}} \\
& \left.\times \exp \left\{-\frac{\left(4^{k} a+\sum_{j=1}^{k-1} 4^{j} a+x-4^{k}(b+c) t\right)^{2}}{2 t}\right\}\right] \\
& \times \exp \left\{-2\left(4^{k-1}\right)(b+c)\right. \\
& \times\left(4^{k} a-\sum_{j=1}^{k-1} 4^{j} a-x-4^{k}(b+c) t\right) \\
& \left.-\frac{4^{2 k-1}(b+c)^{2}}{2} t\right\} \\
& \times \exp \left\{2\left(4^{2 k-1}\right) a(b+c)+2\left(\sum_{j=1}^{k-1} 4^{j} a+x\right) 4^{k-1}(b+c)\right. \\
& \left.-4^{2 k}(b+c)^{2} t\right\} \\
& \times \exp \left\{4^{k}(b+c)\left(\sum_{j=1}^{k-1} 4^{j} a+x\right)\right. \\
& \left.-\frac{9\left(4^{2 k-1}\right)(b+c)^{2}}{2} t\right\} d t
\end{aligned}
$$

For $x>0, a \geq 0$ we have

$$
\begin{aligned}
& \int_{0}^{\infty} \frac{x}{\sqrt{2 \pi t^{2}}} \exp \left\{-\frac{x^{2}}{2 t}-a t\right\} d t \\
& \quad=e^{-x \sqrt{2 a}} \int_{0}^{\infty} \frac{x}{\sqrt{2 \pi t^{2}}} \exp \left\{-\frac{(x-\sqrt{2 a} t)^{2}}{2 t}\right\} d t \\
& =e^{-x \sqrt{2 a}}
\end{aligned}
$$

Besides this estimation, we further have

$$
\mathbb{E}_{x}\left[T_{1} I_{\left[T_{1}<T_{2}\right]}\right]=-\frac{\partial}{\partial x} \cdot \mathbb{E}\left[e^{-a T_{1}} I_{\left[T_{1}<T_{2}\right]}\right] .
$$

\section{Conclusions}

The distribution of first exit time of Brownian motion from a linear barrier has already been investigated in much literature, and most presented results concentrated on a more sophisticated background; the mathematical formulation present in existed literature are usually complicated than the one in this paper. To our best knowledge, in most cases, the distribution is a solution of ODE or PDE, likely did by [1315], and no analytical solution is available. The motivation of this paper is practical validity for applications in risk analysis and financial default probability, and our results principally provide a way to compute the distribution of first exit time of BM from a double linear time-dependent barrier by expressing the aforementioned distribution by infinite series. Although the computer program seems to be complicated, it is still available.

\section{Acknowledgments}

The authors are very grateful to anonymous referees' detailed comments and suggestions, which made this paper much better. $\mathrm{Xu}$ Lin would like to acknowledge the support of the National Natural Science Foundation of China (Grant nos. 11126238 and 11201006) and Humanities and Social Sciences Fund of Ministry of Education, China (Grant no. 12YJC910012). Zhu Dongjin would like to acknowledge the support of the National Natural Science Foundation of China (11271020) and Major Projects of Colleges and Universities in Anhui Province Natural Science Foundation (KJ2012ZD01).

\section{References}

[1] M. Avellaneda and J. Zhu, "Distance to default," Risk, vol. 14, no. 12, pp. 125-129, 2001.

[2] C. Merton, "On the pricing of corporate debt: the risk structure of interest rates," Journal of Finance, vol. 29, pp. 449-470, 1974.

[3] F. Black and J. Cox, "Valuing corporate securities: some effects of bond indenture provisions," Journal of Finance, vol. 31, no. 2, pp. 351-367, 1976.

[4] J. Hull and A. White, "Valuing credit default swaps II: modeling default correlations," Journal of Derivatives, vol. 8, pp. 12-22, 2001.

[5] J. Chadam, L. Cheng, X. Chen, and D. Saunders, "Analysis of an inverse first passage problem from risk management," SIAM Journal on Mathematical Analysis, vol. 38, no. 3, pp. 845-873, 2006

[6] C. Merton, "Option pricing when the underlying stock returns are discontinuous," Journal of Financial Economics, vol. 3, pp. 125-144, 1976.

[7] S. Metwally and A. Atiya, "Using Brownian bridge for fast simulation of jump-diffusion processes and barrier options," The Journal of Derivatives, vol. 10, pp. 43-54, 2002.

[8] X. Zhang, "Numerical analysis of american option pricing in a jump-diffusion model," Mathematics of Operations Research, vol. 22, no. 3, pp. 668-690, 1997.

[9] J. Abate and W. Whitt, "The Fourier-series method for inverting transforms of probability distributions," Queueing Systems, vol. 10, no. 1-2, pp. 5-87, 1992.

[10] H. Gerber and E. S. W. Shiu, "Optimal dividends: analysis with Brownian motion," North American Actuarial Journal, vol. 8, no. 1, pp. 1-20, 2004. 
[11] V. Linetsky, "Lookback options and diffusion hitting times: a spectral expansion approach," Finance and Stochastics, vol. 8, no. 3, pp. 373-398, 2004.

[12] R. DeBlassie, "The lifetime of conditioned Brownian motion in certain Lipschitz domains," Probability Theory and Related Fields, vol. 75, no. 1, pp. 55-65, 1987.

[13] W. Li, "The first exit time of a Brownian motion from an unbounded convex domain," The Annals of Probability, vol. 31, no. 2, pp. 1078-1096, 2003.

[14] M. Lifshits and Z. Shi, "The first exit time of Brownian motion from a parabolic domain,” Bernoulli, vol. 8, no. 6, pp. 745-765, 2002.

[15] D. Lu and L. Song, "The first exit time of a Brownian motion from the minimum and maximum parabolic domains," Journal of Theoretical Probability, vol. 24, no. 4, pp. 1028-1043, 2011.

[16] B. Oksendal, Stochastic Differential Equations and Their Applications, World Scientific Press, New York, NY, USA, 6th edition, 2005.

[17] I. Karatzas and S. Shreve, Brownian Motion and Stochastic Calculus, Springer, New York, NY, USA, 1988. 


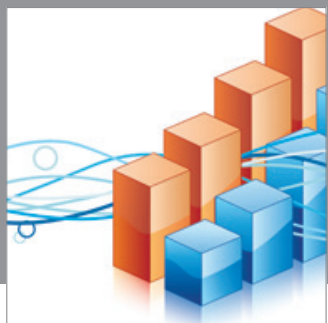

Advances in

Operations Research

mansans

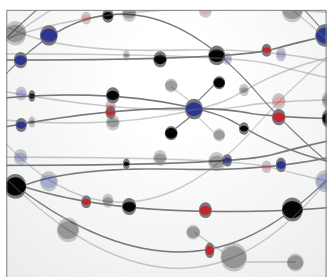

The Scientific World Journal
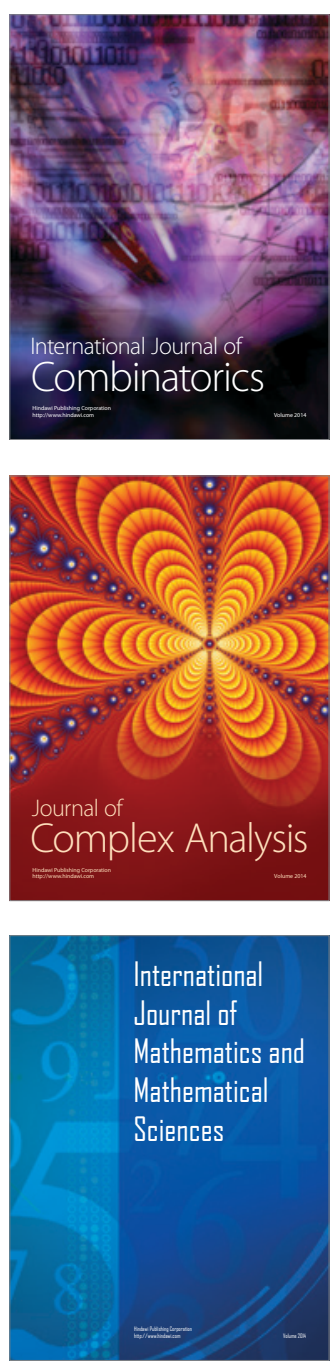
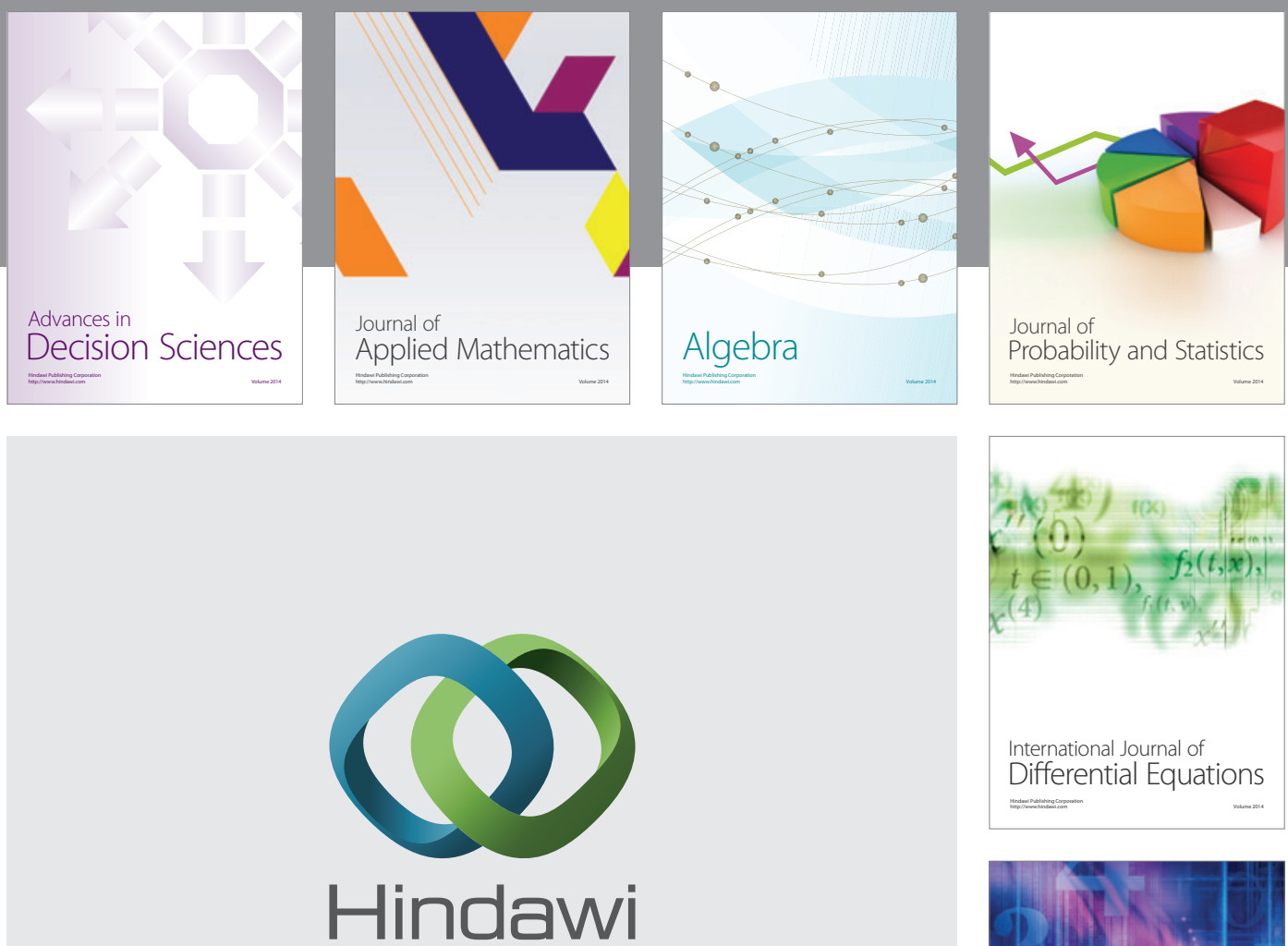

Submit your manuscripts at http://www.hindawi.com
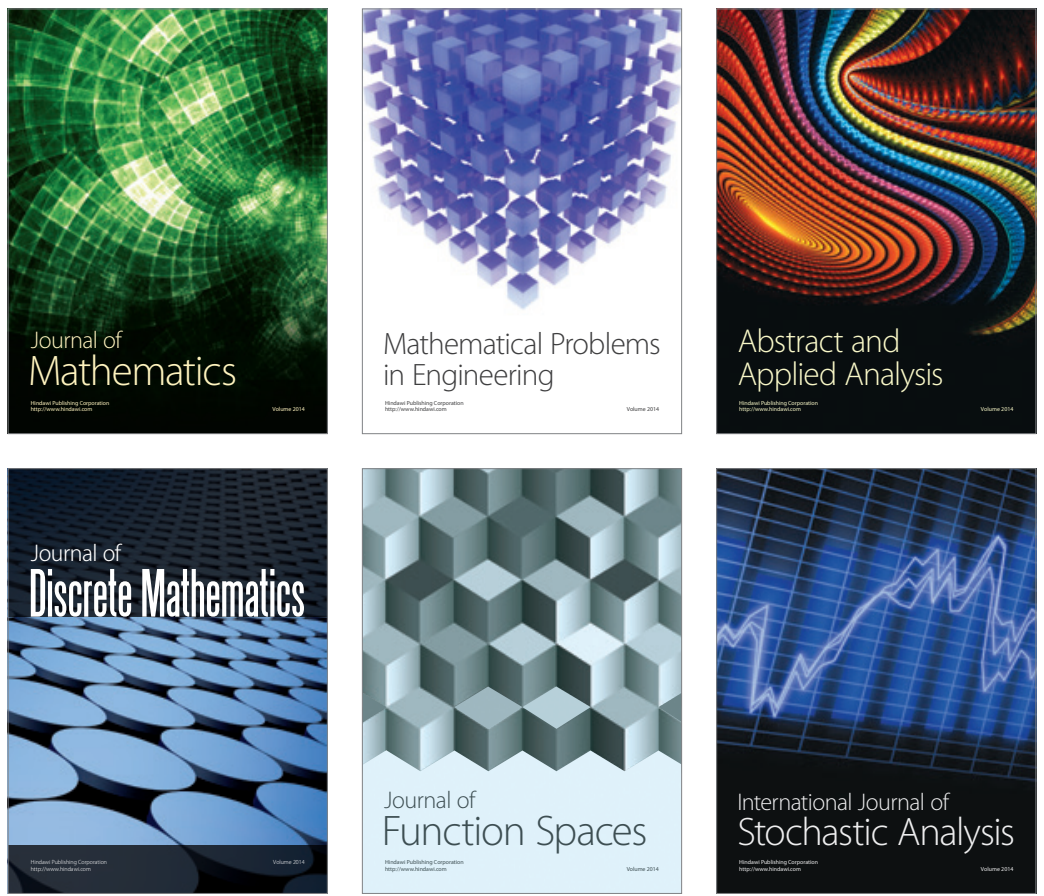

Journal of

Function Spaces

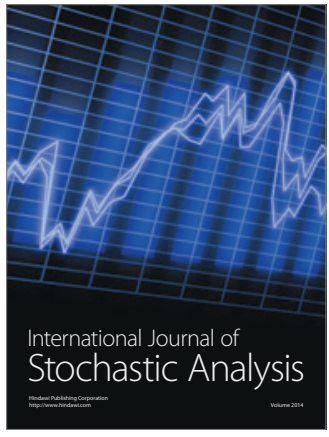

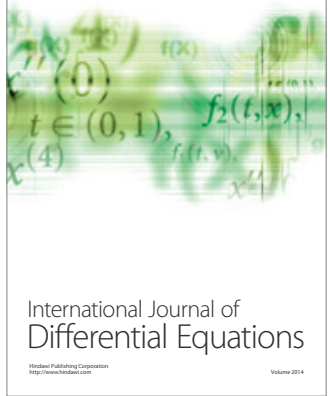
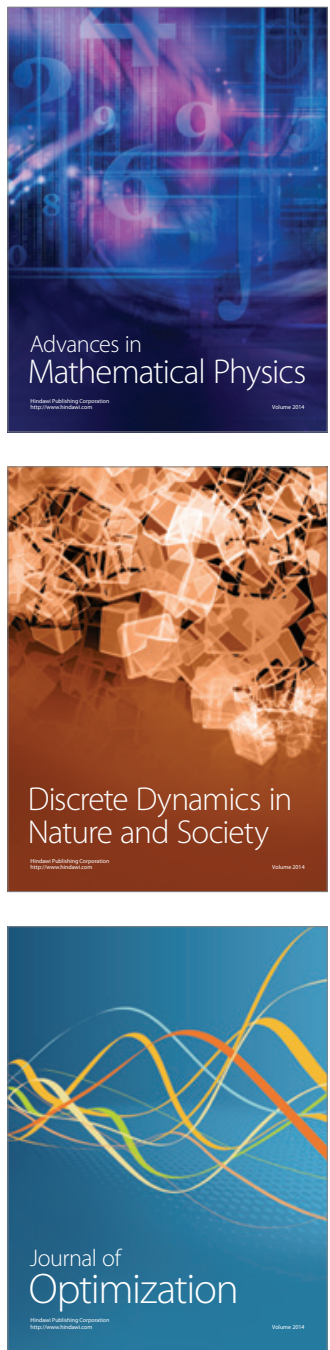